\title{
Avaliações sensoriais, bioquímicas e microbiológicas do guacamole, um produto à base de abacate, sob armazenamento a frio e com adição de ácido ascórbico

\author{
Sensorial, biochemical and microbiological evaluations of \\ guacamole, an avocado based product, under cold storage and \\ added with ascorbic acid
}

\author{
Érica Regina Daiuto ${ }^{*}$; Rogério Lopes Vieites ${ }^{2}$; Juliana Wagner Simon³ Lídia \\ Raquel de Carvalho ${ }^{4}$; Cássia Pegoretti ${ }^{5}$
}

\begin{abstract}
Resumo
A elaboração de produtos de abacate para comercialização mantendo suas características de produto fresco é limitada, já que o fruto cortado escurece rapidamente e suas características sensoriais são modificadas com o armazenamento. Na presente pesquisa, avaliaram-se os parâmetros sensoriais, estabilidade microbiológica e atividade de peroxidase (POD, EC 1.11.1.7) e polifenoloxidase (PPO, EC 1.10.3.1) do guacamole adicionado de ácido ascórbico e conservado sob frio elaborado, usandose abacate da variedade Hass. Produtos acondicionados em embalagens de polietileno+nylon com e sem aplicação de vácuo foram submetidos ao congelamento lento e rápido $\left(-18^{\circ} \mathrm{C}\right)$ e armazenados em freezer $\left(-18^{\circ} \mathrm{C}\right)$. Avaliações foram realizadas no momento da elaboração do produto (t0) e aos 3,7 e 30 dias após armazenamento, sendo que no t30 as amostras foram mantidas sob refrigeração $\left(4 \pm 1^{\circ} \mathrm{C}\right)$ e avaliadas após 3,5 e 7 dias. Após 30 dias de armazenamento, a $-18^{\circ} \mathrm{C}$ sob congelamento, seguido de descongelamento e mantendo-se à $4^{\circ} \mathrm{C} \pm 1$ por 7 dias, as notas para os parâmetros sensoriais diminuram. A peroxidase foi totalmente inibida no produto elaborado e a atividade da enzima polifenoloxidase diminuiu consideravelmente no guacamole $(20,07 \mathrm{mM}$ de catecol/g massa fresca) em relação ao fruto $(58,31 \mathrm{mM}$ de catecol/g massa fresca), porém sem variação significativa durante o armazenamento $\left(\right.$ à $-18^{\circ} \mathrm{C}$ ). As amostras apresentaram-se microbiologicamente estáveis nas condições do estudo. A adição de acido ascórbico contribuiu para conservação do produto de abacate congelado diminuindo a atividade enzimática. No entanto, os parâmetros sensoriais são prejudicados com o descongelamento e armazenamento à $4 \pm 1^{\circ} \mathrm{C}$.
\end{abstract}

Palavras-chave: Persea americana Mill. Refrigeração. Congelamento. Processamento. Parâmetros sensoriais. Peroxidase. Polifenoloxidase.

\begin{abstract}
The elaboration of avocado products for commercialization keeping their characteristics of fresh product has been limited. The cut avocado darkens quickly and their sensorial characteristics are modified with the storage. In the present research, the sensorial parameters, microbiological stability,
\end{abstract}

${ }^{1}$ Pós Doutoranda no curso de Horticultura, Universidade Estadual de São Paulo, FCA/UNESP, Botucatu. C P: 237. E-mail: erdaiuto@uol.com.br

${ }^{2}$ Prof. Dr. Departamento de Gestão e Tecnologia de Alimentos, Universidade Estadual de São Paulo, FCA/UNESP- Botucatu. E-mail: vieites@fca.unesp.br

${ }^{3}$ Aluna de Doutorado no curso Energia na Agricultura da Universidade Estadual de São Paulo, FCA/UNESP- Botucatu. E-mail: julianasimon@fca.unesp.br

${ }^{4}$ Prof $^{a}$ Dra . Departamento de Bioestatística, Universidade Estadual de São Paulo, IB/UNESP- Botucatu. E-mail: lidiarc@ibb.unesp. br

${ }^{5}$ Aluna de Graduação do curso de Nutrição, Universidade Estadual de São Paulo, UNESP- Botucatu. E-mail: ca06so@yahoo.com. br

* Autor para correspondência 
and peroxidase and polyphenoloxidase activity were evaluated in guacamole added with ascorbic acid and conserved under low temperature, by using avocado variety Hass. Products were conditioned in polyethylene+nylon packages with and without vacuum application; then, they were subjected to the slow and fast freezing $\left(-18^{\circ} \mathrm{C}\right)$ and stored in freezer $\left(-18^{\circ} \mathrm{C}\right)$. Evaluations were performed at the moment of elaboration of the product ( $\mathrm{t} 0)$ and at 3,7 and 30 days post-storage. At $\mathrm{t} 30$, samples were kept under refrigeration $\left(4 \pm 1^{\circ} \mathrm{C}\right)$ and evaluated at 3,5 and 7 days. After the 30 days of storage, -18 ${ }^{\circ} \mathrm{C}$ under freezing, followed by thawing and keeping at $4 \pm 1^{\circ} \mathrm{C}$ for 7 days, the notes for the sensorial parameters decreased. The peroxidase activity was totally inhibited in the elaborated product and the polifenol oxidase activity considerably decreased in the guacamole $(20.07 \mathrm{mM}$ catechol/g fresh matter) relative to those in the fruit ( $58.31 \mathrm{mM}$ catechol/g fresh matter), however with no significant variation during storage (at $-18^{\circ} \mathrm{C}$ ). The samples were microbiologically stable under the conditions of the present study. The addition of ascorbic acid contributed to the conservation of the frozen avocado product by decreasing the enzymatic activity. However, the sensorial parameters are prejudiced under thawing and storage at $4 \pm 1^{\circ} \mathrm{C}$.

Key words: Persea americana Mill. Refrigeration. Freezing. Processing. Sensorial parameters. Peroxidase. Polyphenol oxidase.

\section{Introdução}

O abacate (Persea americana Mill.) é uma fruta tropical, sendo que no Brasil as variedades Hass e Fuerte são comercializadas inclusive no mercado internacional, com selo de certificação, sob a denominação Avocado.

As qualidades sensoriais, o valor nutritivo e a riqueza vitamínica do abacate justificam plenamente a expansão de seu consumo. No Brasil, o fruto é consumido com açúcar, mas nos outros países é consumido na forma de salada, sopa e molho, a exemplo do guacamole, preparado com polpa de abacate (não liquefeita), suco de limão e cebola. O guacamole pode consistir uma alternativa de comercialização para frutos sem selo de certificação ou refugos da produção.

É um desafio a comercialização do abacate na forma processada, pois a polpa do abacate é altamente sensível ao escurecimento pela ação da polifenoloxidase (PFO - o-difenol: oxigênio oxidoredutases: E.C. 1.10.3.1) e reações de degradação pela ação da peroxidase (POD - doador: $\mathrm{H}_{2} \mathrm{O}_{2}$ oxidoredutase, EC 1.11.1.7), além do alto teor de matéria graxa no fruto, que o torna susceptível à rancidez oxidativa e hidrolítica (BATES, 1970 apud MARTIN, 1991).

Desde 1915, diversos países tentam estabilizar a polpa de abacate, empregando preservação sob pasteurização, secagem, extração de óleo, congelamento e liofilização (MARTIN, 1991). O congelamento adequado permite a conservação do sabor e textura, praticamente sem alteração, por mais de um ano (RUELHLE, 1974 apud MARTIN, 1991). Já as temperaturas de pasteurização desenvolvem sabores estranhos e mudanças consideráveis na cor do produto (CRUESS et al. apud STEPHENS; LIME; GRIFFITHS, 1958). Guzmán et al. (2002) avaliaram o uso do aquecimento em microondas aliado ao uso de cloreto de cobre na preservação da cor do purê de abacate. Usam-se também agentes químicos redutores, sequestrantes e ácidos (SON; MOON; LEE, 2000). López-Malo et al. (1998) sugere que estas substâncias podem ser usadas em processos combinados como tratamentos sob alta pressão. Palou et al. (1999, 2000) obtiveram resultados significativos na diminuição da atividade das enzimas polifeonoloxidase e lipoxigenase, responsáveis por alterações no produto processado, com o tratamento sob alta pressão.

Das opções apresentadas na literatura para evitar o escurecimento enzimático no guacamole, as mais utilizadas na prática, consistem no tratamento térmico e a utilização de aditivos. O tratamento térmico inativa a PFO, mas pode alterar a granulosidade do guacamole, tornando-o com aparência de um purê ou pasta, além do desenvolvimento de gosto amargo (BEN-ET; DOLEV; TATARSK, 1973). 
Na presente pesquisa, avaliaram-se os parâmetros sensoriais, estabilidade microbiológica e atividade das enzimas PFO e POD em guacamole elaborado com adição de ácido ascórbico e conservado pelo frio, visando preservação de qualidades sensoriais e nutritivas do produto.

\section{Material e Métodos}

\section{Matéria prima}

Abacates da variedade Hass (avocado) foram fornecidos pela empresa Jaguacy, São Paulo. Molho de pimenta, sal, limão, cebola, limão Taiti e tomate foram obtidos em supermercado local.

\section{Processamento}

Os abacates amadurecidos foram processados manualmente seguindo as boas práticas de manipulação de alimentos para obter o guacamole. Os frutos foram previamente armazenados à $4 \pm 1^{\circ} \mathrm{C}$ para retardar ou prevenir o escurecimento, conforme procedimento utilizado para alimentos minimamente processados. Os tomates, cebolas e limões utilizados foram sanitizados da mesma forma que os frutos de abacate. A formulação utilizada para o guacamole foi: $500 \mathrm{~g}$ de abacate, $20 \mathrm{~g}$ de limão, 7,5 $\mathrm{g}$ molho de pimenta, $15 \mathrm{~g}$ de cebola, $70 \mathrm{~g}$ de tomate, 5,5 g de sal e 12,6 g de ácido ascórbico.

\section{Tratamentos}

O guacamole foi armazenado em embalagens de nylon + polietileno previamente irradiadas com 10 kGy. Posteriormente, foi submetido ao congelamento lento por $6 \mathrm{~h} \mathrm{a}-18^{\circ} \mathrm{C}$ e congelamento rápido por 30 min a $-18^{\circ} \mathrm{C}$ (Irinox-refrigerador HCFC 22, marca local), e então foi armazenado em freezer doméstico $\left(-18^{\circ} \mathrm{C}\right)$. As avaliações foram realizadas no dia da elaboração do produto (to) e as amostras congeladas foram analisadas após 3, 5, 7 e 30 dias. Amostras t30 foram descongeladas e mantidas a $4 \pm 1 \mathrm{oC}$ e avaliadas aos 3, 5 e 7 dias, simulando a condição de uso do produto. As análises foram realizadas até que a qualidade fosse aceitável para o consumidor, considerando o aspecto visual e coloração.

\section{Análise sensorial}

A avaliação sensorial foi realizada utilizando-se o método de aceitação (CHAVES; SPROESSER, 1999) em condições laboratoriais com um grupo de 30 provadores não treinados escolhidos ao acaso. O delineamento utilizado foi o de blocos ao acaso. Foram aplicadas fichas indicando os parâmetros sensoriais aparência, cor, textura, sabor e aceitação. Os provadores foram orientados a marcar com um traço vertical na escala de 9 pontos, e as notas de cada parâmetro foram utilizadas para análise estatística dos dados. As avaliações foram realizadas às $10 \mathrm{ou}$ às $15 \mathrm{~h}$ e as amostras servidas com Dipas, salgadinho de milho comumente usado para acompanhar guacamole.

\section{Atividade da peroxidase (POD, EC 1.11.1.7)}

A extração e a determinação da POD foram realizadas de acordo com o método adaptado de Lima (1994). Três gramas de polpa congelada em $\mathrm{N}$ líquido e armazenada em freezer foram homogeneizados em $30 \mathrm{~mL}$ de tampão acetato de sódio $100 \mathrm{mM}$ pH 5,0 e centrifugados a $12.000 \mathrm{x} \mathrm{g}$ por $25 \mathrm{~min}$ a $4^{\circ} \mathrm{C}$. Em seguida, a amostra foi filtrada e tomou-se o sobrenadante límpido, com o qual foi feita a determinação da atividade da peroxidase em tubos de ensaio contendo $1 \mathrm{~mL}$ de extrato bruto, 0,5 $\mathrm{mL}$ de sol.A $\left(2,2 \mathrm{~mL} \mathrm{H}_{2} \mathrm{O}_{2}\right.$ A $30 \%$ em $10 \mathrm{~mL}$ de água em tampão fosfato $\mathrm{pH}$ 6,7) e $0,5 \mathrm{~mL}$ de sol.B (81,5 mg de fenol em $40 \mathrm{~mL}$ de $\mathrm{H}_{2} \mathrm{O}$, dissolvidos em 40,65 mg de amino anti pirina (AAP) em 10 $\mathrm{mL}$ de $\mathrm{H}_{2} \mathrm{O}$ ), colocados em banho-maria por $5 \mathrm{~min}$ a $30^{\circ} \mathrm{C}$ sob agitação. Após $5 \mathrm{~min}$, adicionaram-se $2 \mathrm{~mL}$ de álcool etílico a fim de parar a reação, e posteriores leituras de absorbância foram realizadas em espectro a $505 \mathrm{~nm}$. 
Atividade da polifenoloxidase (PPO, EC 1.10.3.1)

Determinada a partir do mesmo extrato bruto obtido no item 5. O sobrenadante foi utilizado para determinar a atividade da enzima PFO (CANO et al., 1997). O sistema de reação consistiu-se de $0,3 \mathrm{~mL}$ de extrato e $1,85 \mathrm{~mL}$ de catecol $0,1 \mathrm{M}$ e permaneceu em banho-maria por $30 \mathrm{~min}$. a $30^{\circ} \mathrm{C}$ sob agitação. Após $30 \mathrm{~min}$, adicionou-se $0,8 \mathrm{~mL}$ de ácido sulfúrico a fim de parar a reação, e posteriores leituras de absorbância foram realizadas em espectrofotômetro a $395 \mathrm{~nm}$.

\section{Avaliações microbiológicas}

As análises seguiram os métodos da American Public Health Association (APHA, 2001).

Preparo das amostras e diluições: $25 \mathrm{~g}$ do produto foram colocados em $225 \mathrm{~mL}$ de água peptonada tamponada previamente esterilizada, com posterior homogeneização em Stomacher Lab Blender 400 por 30s. Após tal diluição $\left(10^{-1}\right)$, foram realizadas novas diluições em tubos de ensaio contendo $9 \mathrm{~mL}$ de salina estéril.

Determinação do Número Mais Provável (NMP) de coliformes totais e termotolerantes: $1 \mathrm{ml}$ da diluição foi inoculado em uma série de 3 tubos de ensaio, com tubos de Durham invertido, contendo $10 \mathrm{~mL}$ de caldo lauril sulfato (Difco), que então foram incubados em estufa a $35{ }^{\circ} \mathrm{C}$ durante $48 \mathrm{~h}$. Tubos que apresentaram turvação e gás (bolhas) dentro dos tubos de Durham foram repicados para outros tubos contendo $5,0 \mathrm{~mL}$ de caldo lactosado bile verde brilhante, encubados a 35 ${ }^{\circ} \mathrm{C}$ por $48 \mathrm{~h}$. Uma outra alíquota foi transferida para tubos com Durhan contendo $5,0 \mathrm{~mL}$ de caldo EC (Difco) e encubados em estufa a $45^{\circ} \mathrm{C}$ por $24 \mathrm{~h}$. A caracterização de teste positivo é turvação do meio e produção de gás nos tubos

Detecção da presença de Salmonella: $1 \mathrm{~mL}$ da amostra diluída foi transferido para tubo de ensaio contendo $10 \mathrm{~mL}$ de caldo tetrationato (TT-Difco) com $0,1 \mathrm{~mL}$ de iodo-iodeto, com posterior incubação em estufa a $35^{\circ} \mathrm{C} / 24 \mathrm{~h}$. Uma outra alíquota de $0,1 \mathrm{~mL}$ foi transferida para tubo de ensaio contendo $10 \mathrm{~mL}$ de caldo Rapapport Vassiliadis (Difco), com posterior incubação em estufa a $42{ }^{\circ} \mathrm{C} / 24 \mathrm{~h}$. Transcorrido os tempos de incubação, uma alçada de cada meio de cultura foi estriada em placas de Petri contendo agar xilose-lisina-desoxicolato (XLD-Difco) e agar Salmonella \& Shigella (SSMERCK). As placas foram incubadas em estufa a $35^{\circ} \mathrm{C} / 24 \mathrm{~h}$. As colônias características de Salmonella foram isoladas e repicadas para tubos de ensaio contendo ágar tripticase soja inclinado (TSAOxoid), sendo consideradas cepas estoques, com posterior incubação a $35^{\circ} \mathrm{C}$ por $24 \mathrm{~h}$. A partir desse crescimento foram feitos repiques em tubos de ensaio contendo ágar tríplice açúcar ferro inclinado (TSI-Difco) e em tubos contendo ágar fenilalanina inclinado (Difco). Os tubos foram incubados em estufa à $35^{\circ} \mathrm{C} / 18-24 \mathrm{~h}$. O teste de TSI positivo para Salmonella deve apresentar base amarela com ou sem produção de gás, evidenciada pela presença de bolhas e com ápice vermelho. Pode ocorrer também a produção de ácido sulfídrico $\left(\mathrm{H}_{2} \mathrm{~S}\right)$, tornando $o$ meio enegrecido. $O$ teste de fenilalanina foi realizado adicionando-se algumas gotas de solução de cloreto férrico $10 \%$ sobre a colônia formada e observando-se a alteração ou não da cor do meio.

Enumeração de Staphylococcus coagulase positiva: utilizou-se o método de semeadura em superfície, onde $0,1 \mathrm{~mL}$ da diluição $10^{-1}$ da amostra de guacamole foi colocado em placa de Petri contendo ágar Baird-Parker (Difco) suplementado com 5\% de telurito de potássio e solução de gema de ovo (Difco). A amostra foi espraiada com auxílio de bastão de vidro em "L". Em seguida, as placas de Petri foram incubadas em posição invertida, a $35^{\circ} \mathrm{C} / 48 \mathrm{~h}$ em estufa.

\section{Enumeração de bactérias do grupo Bacillus} cereus: realizou-se semeadura em superfície, onde $0,1 \mathrm{~mL}$ da amostra foi depositado na superfície do ágar gema de ovo polimixina vermelho de fenol e espraiada com uma alça em L. As placas foram 
incubadas à $35^{\circ} \mathrm{C} / 24 \mathrm{~h}$ e, em seguida, foram contadas as placas que apresentaram entre 25-250 colônias características, sendo róseas e irregulares, com halo de precipitação, devido a ação da lecitinase.

\section{Análise estatística}

Os resultados da análise sensorial foram avaliados por meio da análise de componentes principais (BARROSO; ARTES, 2003). Baseado nos escores produzidos pela aplicação destes componentes a cada amostra, procedeu-se a análise de agrupamentos (BARROSO; ARTES, 2003), visando detectar grupos de amostras com avaliações semelhantes. As análises estatísticas foram executadas com o software R (R DEVELOPMENT CORE TEAM, 2007).

\section{Resultados e Discussão}

Avaliação sensorial

Daiuto et al. (2007) avaliaram a qualidade sensorial do guacamole produzido com a variedade
Hass sem a presença de aditivos químicos, acondicionado em embalagens de polietileno e de polietileno+nylon e submetido ao tratamento frio: refrigeração e congelamento lento e rápido, sob as mesmas condições utilizadas no presente estudo e obtiveram notas melhores (de 7 a 9) para os parâmetros sensoriais avaliados em embalagens de nylon+polietileno congeladas, sendo que as piores avaliações foram para embalagens de polietileno sob tratamento refrigerado. Por este motivo, no presente estudo só foi utilizada a embalagem de nylon + polietileno.

A (Figura 1) apresenta os gráficos de dispersão para cada par de variáveis sensoriais, permitindo extrair evidências relativas às associações existentes entre tais variáveis.

As avaliações de textura e cor parecem apresentar relação linear positiva. À medida que aumenta o conceito atribuído à cor, o conceito atribuído à textura aumentou linearmente. Comportamento semelhante pode ser verificado para outros pares de variáveis, como cor e sabor ou aceitação e sabor, por exemplo.

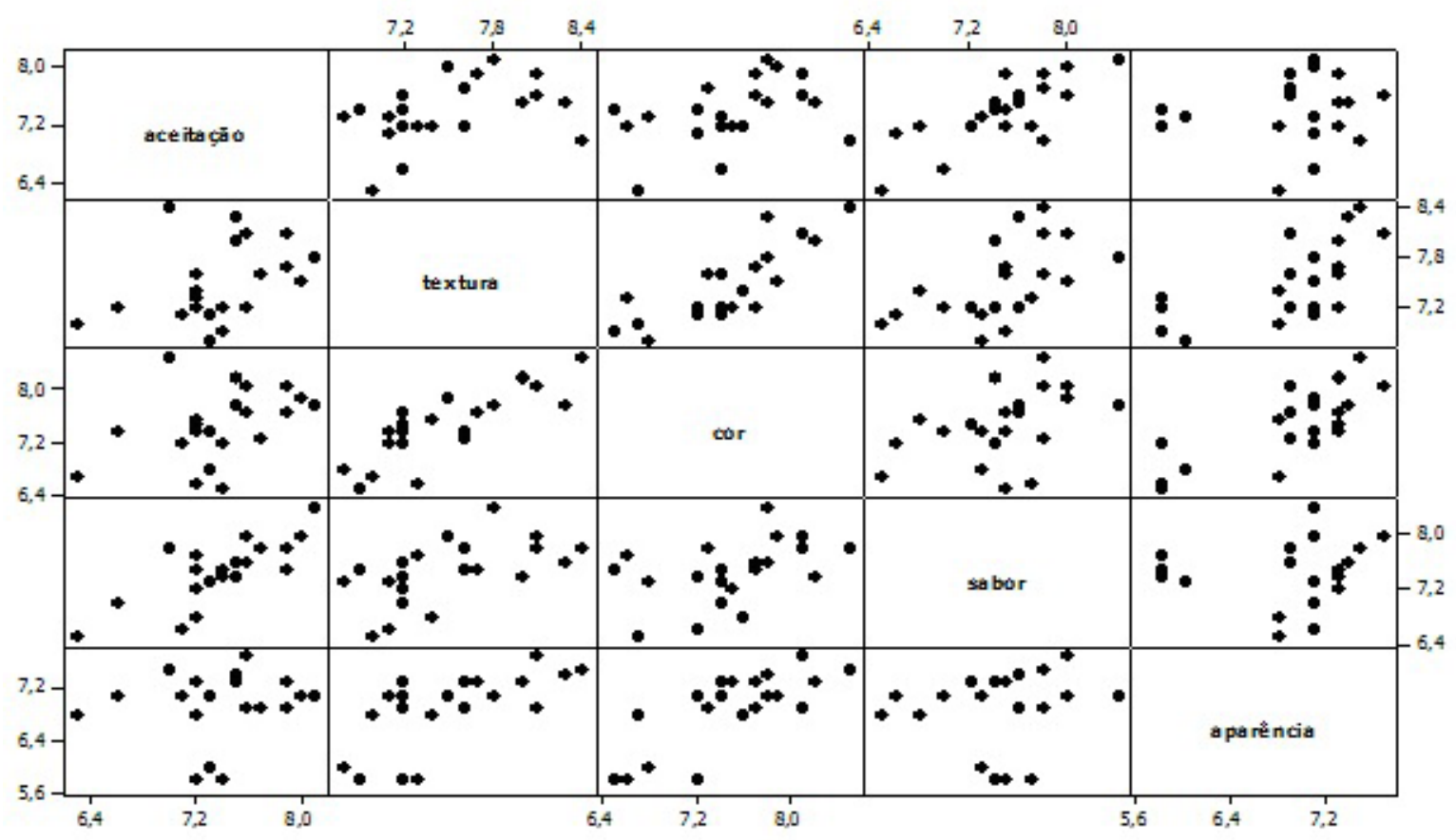

Figura 1. Gráficos de dispersão para os parâmetros da análise sensorial. 
Tabela 1. Correlações lineares para os parâmetros da análise sensorial.

\begin{tabular}{lcclll}
\hline & Aceitação & Textura & Cor & Sabor & Aparência \\
\hline Aceitação & 1,00 & 0,39 & $0,42^{*}$ & $0,77^{*}$ & 0,10 \\
Textura & 0,39 & 1,00 & $0,83^{*}$ & $0,56^{*}$ & $0,63^{*}$ \\
Cor & $0,42^{*}$ & $0,83^{*}$ & 1,00 & $0,44^{*}$ & $0,76^{*}$ \\
Sabor & $0,77^{*}$ & $0,56^{*}$ & $0,44^{*}$ & 1,00 & 0,13 \\
Aparência & 0,10 & $0,63^{*}$ & $0,76^{*}$ & 0,13 & 1,00 \\
\hline
\end{tabular}

$*(\mathrm{P}<0,05)$

As correlações entre aceitação e textura e aceitação e aparência foram as únicas não significativas $(\mathrm{p}<0,5)$. $\mathrm{Na}$ (Tabela 1) observa-se que houve também correlações moderadas ( $\mathrm{r}$ entre 0,40 e 0,69 ) e correlações fortes (entre 0,69 e 0,89$)$. Os maiores valores de correlação são para textura e cor, sabor e aceitação, aparência e cor. A melhor avaliação para a cor da amostra refletiu nos parâmetros de textura e aparência, assim como o melhor sabor na aceitação do produto.

A (Tabela 2) apresenta um resumo quanto à importância dos componentes na explicação da variação dos dados. Verifica-se que os dois primeiros componentes são capazes de conservar $87 \%$ da variação original dos dados, quantidade bastante expressiva, viabilizando o prosseguimento da análise com base nestas duas novas variáveis.

Tabela 2. Porcentagem da variância dos dados com base em cada um dos cinco componentes obtidos.

\begin{tabular}{llllll}
\hline & \multicolumn{5}{c}{ Componente } \\
\cline { 2 - 6 } & $\mathbf{1}$ & $\mathbf{2}$ & $\mathbf{3}$ & $\mathbf{4}$ & $\mathbf{5}$ \\
\hline Variância explicada (\%) & 0,61 & 0,26 & 0,07 & 0,04 & 0,02 \\
Variância explicada acumulada (\%) & 0,61 & 0,87 & 0,94 & 0,98 & 1,00 \\
\hline
\end{tabular}

As funções apresentadas na seqüência expressam os dois componentes principais. A análise de seus coeficientes permite avaliar as correlações existentes entre as variáveis sensoriais.

\section{- Componente 1:}

$$
C P 1=0,38^{*} \text { aceitaçäo }+0,51^{*} \text { textura }+0,51^{*} \text { cor }+0,41^{*} \text { sabor }+0,39 * \text { aparência }
$$

O primeiro componente atribui ponderações positivas e de magnitudes semelhantes a cada uma das variáveis sensoriais consideradas, evidenciando correlações positivas entre as cinco variáveis sob estudo. Amostras de guacamole bem apreciadas no conjunto de variáveis sensoriais produzirão valores positivos com magnitudes grandes para este componente, enquanto amostras mal apreciadas fornecerão resultados negativos.

\section{Componente 2:}

$$
C P 2=0,57 * \text { aceitação }-0,16 * \text { textura }-0,26 * \text { cor }+0,53 * \text { sabor }+0,39 * \text { aparência }
$$


Observando as ponderações associadas a cada variável neste componente, tem-se que ele contrapõe a aparência das amostras à cor e à textura. Amostras com valores elevados para este componente têm aparência bem avaliada, com menores notas para a cor e a textura. Amostras com valores reduzidos para o segundo componente têm avaliações inversas às citadas anteriormente.

Para cada amostra de guacamole, foram calculados os respectivos escores para os dois componentes considerados. Com base nos escores obtidos, realizou-se uma análise de agrupamentos (BARROSO; ARTES, 2003) com o objetivo de detectar amostras de guacamole com avaliações semelhantes quanto às variáveis sensoriais (Figura 2). O método de agrupamento utilizado foi o de Ward e optou-se, com base no dendograma obtido, pela composição de seis clusters.

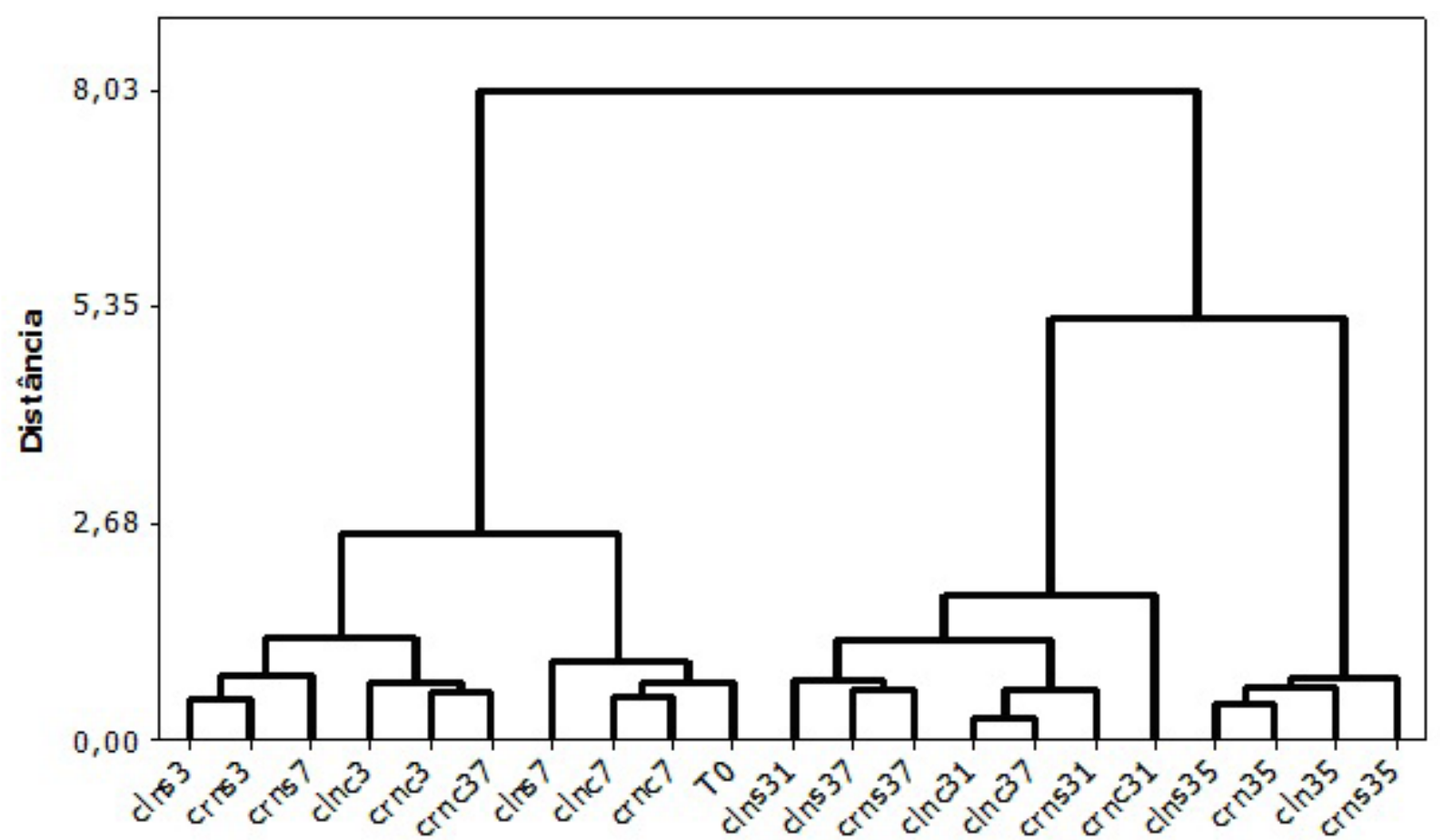

Tratamento frio: $\mathrm{CL}=$ congelamento lento, $\mathrm{CR}=$ congelamento rápido; Embalagens: $\mathrm{NS}=$ sem vácuo, $\mathrm{NC}$ : com vácuo; Dias de armazenamento: 3, 7, 31,35 e 37 dias; T0= tempo zero.

Figura 2. Dendograma da análise de agrupamentos baseada nos escores calculados sob os dois componentes principais, para cada amostra de guacamole.

A (Figura 3) apresenta os escores das amostras de guacamole em relação aos dois componentes iguais pertencem a um mesmo grupo, dentre os sete principais. Amostras representadas por símbolos fornecidos pela análise de agrupamentos. 


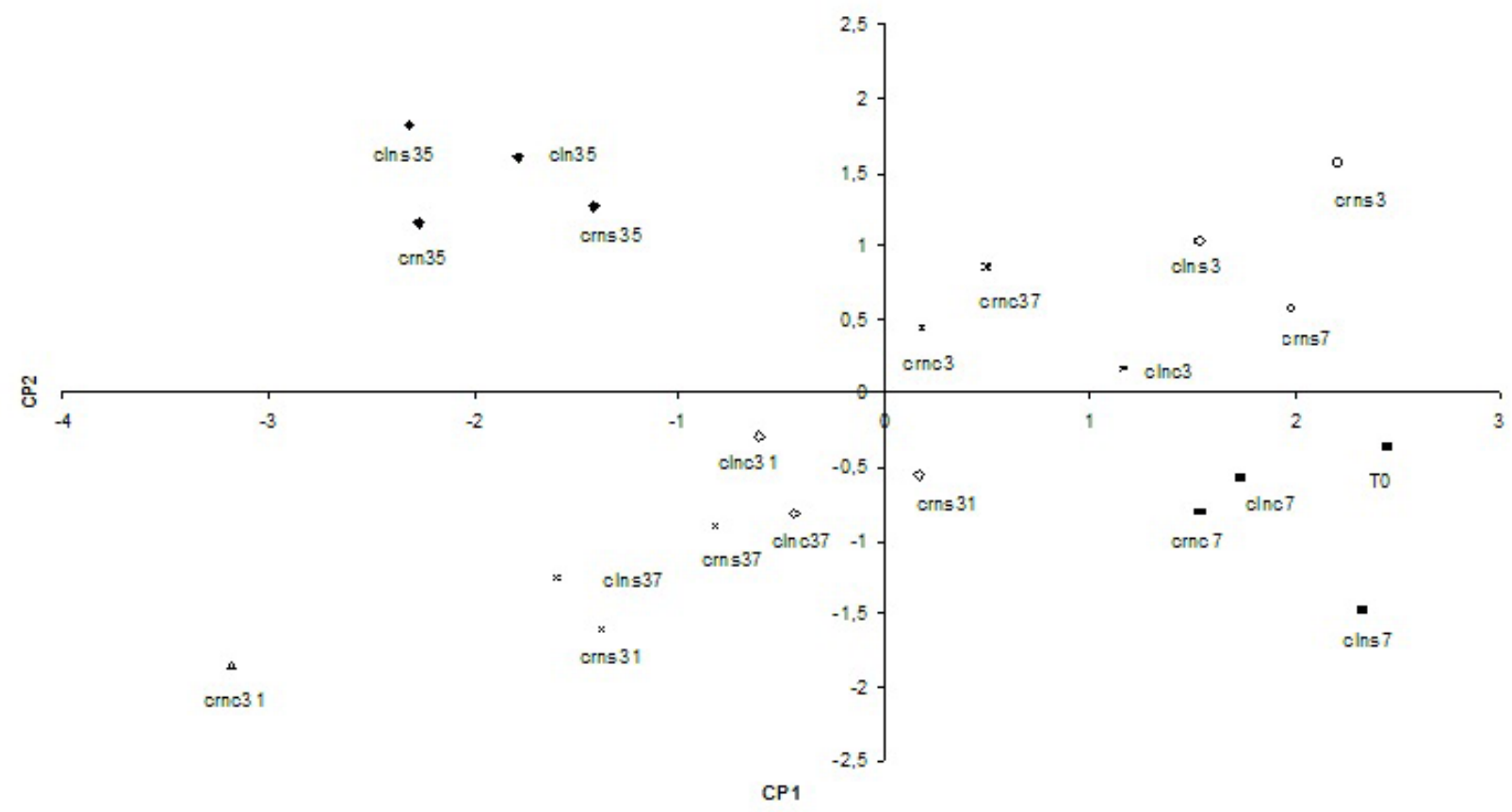

Tratamento frio: $\mathrm{CL}=$ congelamento lento, $\mathrm{CR}=$ congelamento rápido; Embalagens: $\mathrm{NS}=$ sem vácuo, $\mathrm{NC}$ : com vácuo; Dias de armazenamento: 3, 7, 31, 35 e 37 dias; T0= tempo zero.

Figura 3. Escores para cada amostra de guacamole e coeficientes para cada variável associados aos dois componentes principais.

O grupo 1 foi formado pelas amostras CLNS3, CRNS3 e CRNS7 e teve o melhor desempenho com as maiores médias para os parâmetros sensoriais avaliados e se destacaram por notas maiores na aceitação do produto $(7,9$ a 8,0$)$. O grupo 2 foi formado pelas amostras CLNC3, CRNC3 e CRNC37 e também teve um bom desempenho, mas com médias um pouco inferiores em relação ao grupo 1 para os parâmetros sensoriais avaliados. Os grupos 1 e 2 compreenderam as amostras mais bem avaliadas armazenadas até o sétimo dia em embalagem de nylon+polietileno com e sem vácuo. Daiuto et al. (2007), ao avaliarem sensorialmente o guacamole sem presença de aditivos e armazenado sob frio em embalagens de polietileno e nylon + polietileno (com e sem vácuo), não verificaram diferenças quanto à aplicação de vácuo, sendo que o efeito da embalagem na conservação do produto foi mais evidente. Os autores constataram um produto de melhor qualidade na embalagem de nylon + polietileno.
O grupo 3 foi formado pelas amostras CLNS7, CLNC7, CRNC7 e T0 e caracterizaram-se pelas maiores notas de textura, cor e sabor $(7,8$ a 8,5$)$.

O grupo 4 foi formado pelas amostras CLNS31 (seria CRNS31?), CLNS37 e CRNS37 e não apresentou bom desempenho como os grupos anteriores, mas também não teve o pior desempenho. O grupo 5, formado pelas amostras CLNC31, CLNC37 e CRNS31, também não teve um bom desempenho. O grupo 6, formado pela amostra CRNC31, teve o pior desempenho. O grupo 7 foi formado pelas amostras CLNS35, CRN35, CLN35 e CRNS35 e teve um desempenho intermediário. Para estes grupos, as notas para os parâmetros avaliados variaram de 6,3 a 7,6.

A partir dos grupos formados, foi possível observar que, após 30 dias de armazenamento sob congelamento, seguido de descongelamento e armazenamento sob refrigeração por 7 dias, ocorre alterações negativas nos parâmetros sensoriais avaliados. 
Atividade das enzimas polifenoloxidase $e$ peroxidase

Não foi identificada no presente estudo nenhuma atividade da peroxidase com a adição de ácido ascórbico. A peroxidase é uma enzima que pode levar à destruição da vitamina $\mathrm{C} \mathrm{e}$ descoloração de carotenóides e antocianinas, além de catalisar a degradação não-enzimática de ácidos graxos insaturados, com formação de compostos voláteis. É considerada uma das enzimas mais termorresistentes, e sua atividade diminui em $\mathrm{pH}$ baixo ou elevado. A perda de atividade observada no presente estudo com a acidificação é atribuída a mudanças de seu estado nativo para a condição desnaturada em função da liberação do grupo heme da proteína. $\mathrm{O} \mathrm{pH}$ ótimo varia com a fonte de 3 a 7 (ARAÚJO, 2006). Neste experimento, a diminuição do $\mathrm{pH}$ pela adição de limão (contido na formulação) e ácido ascórbico pode ter resultado na inibição da atividade da peroxidase.

A polifenoloxidase é uma das enzimas responsáveis pelo escurecimento do abacate. $\mathrm{Na}$ ausência do ácido ascórbico, a enzima modifica os compostos fenólicos presentes nas células (ácido clorogêncio, leucoantocianinas, catecol e outros). O rompimento das paredes celulares deixa os mencionados compostos em contato com as enzimas, que catalisam sua oxidação a compostos do tipo quinona, até a transformação em melaninas de intensa coloração marrom. As melaninas, embora não tóxicas, alteram a aparência do produto, além de induzir mudanças no aroma e no sabor (MARTIN, 1991).

A atividade da polifenoloxidase no fruto, antes da elaboração do produto, foi de $58,31 \mathrm{mM}$ de catecol/g massa fresca. A atividade no guacamole no dia da elaboração do produto foi de $20,07 \mathrm{mM}$ de catecol/g massa fresca. Nota-se já no produto elaborado uma queda na atividade da enzima. O ácido ascórbico é um antioxidante sinergístico e removedor de oxigênio. Age diretamente com o oxigênio formando o deidroacético ascórbico, e assim elimina o suprimento de oxigênio disponível a reações de auto-oxidação (ARAÚJO, 2006). Na formulação do guacamole, é incorporado ácido cítrico, um constituinte natural do limão. Na indústria de alimentos, o ácido cítrico é um acidificador, flavorizante, sequestrante e tamponante, sendo usado como sinergista a antioxidantes primários e removedores de oxigênio (ARAÚJO, 2006).

Nos tratamentos sem aplicação de vácuo (Figura 4 a), a atividade da enzima variou de 8,1 a $9,6 \mathrm{mM}$ de catecol/g massa fresca no congelamento rápido, e de 8,1 a $9,53 \mathrm{mM}$ de catecol/g massa fresca no congelamento lento, a partir do tempo 3. Já nos tratamentos com aplicação de vácuo (Figura 4 a), a atividade foi de 7,2 a $13,7 \mathrm{mM}$ de catecol/g massa fresca para o congelamento rápido e de 7,9 a 16,47 $\mathrm{mM}$ de catecol/g massa fresca para o congelamento lento. 

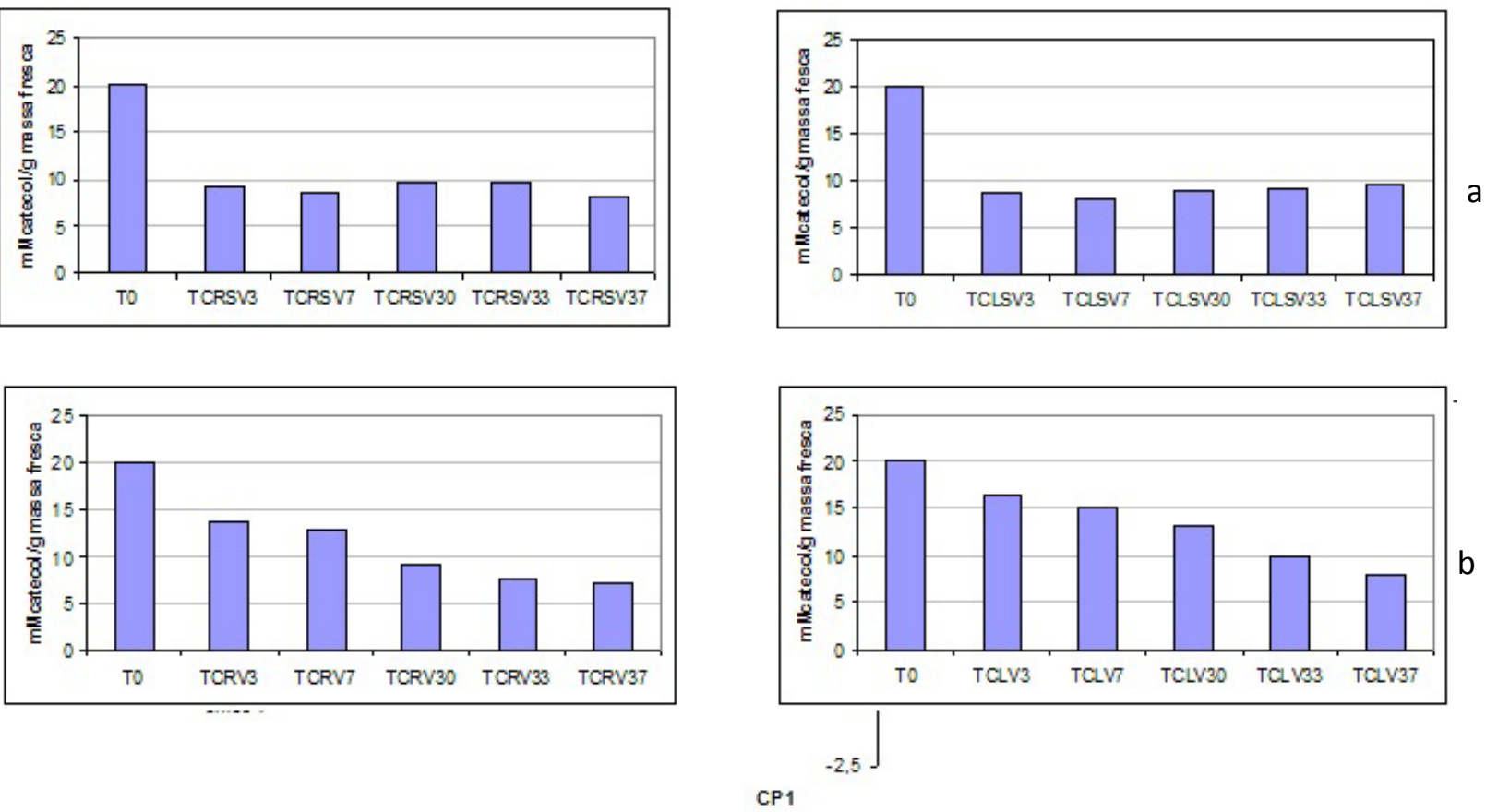

$\mathrm{TCL}=$ congelamento lento, $\mathrm{TCR}=$ congelamento rápido; Embalagens: $\mathrm{SV}=$ sem vácuo, V: com vácuo; Dias de armazenamento: $3,7,31,35$ e 37 dias; $\mathrm{T} 0=$ tempo zero.

Figura 4. Atividade da enzima polifenoloxidase em amostras de guacamole armazenadas em embalagem de nylon+polietileno. a) sem aplicação de vácuo e b) com aplicação de vácuo.

$\mathrm{Na}$ (Figura 4), observa-se tendência de diminuição da atividade da enzima após 30 dias de armazenamento congelado e posterior refrigeração. No tratamento a vácuo, após 30 dias também se verifica diminuição na atividade da enzima.

O armazenamento por congelamento ou refrigeração levou à pouca alteração na atividade da polifenoloxidase do guacamole no armazenamento, mas observou-se tendência de diminuição de sua atividade na estocagem nos tratamentos com aplicação de vácuo.

\section{Avaliações microbiológicas}

Os resultados do presente estudo foram comparados aos parâmetros estabelecidos pela Agência Nacional de Vigilância Sanitária RDC $\mathrm{n}^{\circ} 12$ de 02 de janeiro de 2001(ANVISA, 2008). De acordo com essa resolução, o produto final deve conter no máximo $102 \mathrm{NMP} / \mathrm{g}$ de coliformes termotolerantes, $103 \mathrm{UFC} / \mathrm{g}$ de Staphylococcus aureus e Bacillus cereus e ser ausente de Salmonella em $25 \mathrm{~g}$ de produto analisado.

O grupo controle apresentou coliformes totais em níveis $>1.100 \mathrm{NMP} / \mathrm{g}$ (Tabela 3 ). Sob congelamento lento com vácuo, observou-se que o guacamole apresentou resultados com valores iguais a 9 $\mathrm{NMP} / \mathrm{g}$ para os dias 5 e 7 de armazenamento. Para o congelamento lento sem vácuo, os resultados obtidos foram 7 e $4 \mathrm{NMP} / \mathrm{g}$ nos dias 5 e 7, respectivamente. No congelamento rápido com vácuo, verificaramse resultados de $9 \mathrm{NMP} / \mathrm{g}$ no dia 5 e $<3,0$ no dia 7 de armazenamento. No congelamento rápido sem vácuo, os resultados obtidos foram $4 \mathrm{NMP} / \mathrm{g}$ e $<$ $3,0 \mathrm{NMP} / \mathrm{g}$ quando armazenados durante 5 e 7 dias respectivamente.

Aos 30 dias de armazenamento, para todos os tratamentos, o guacamole apresentou valores $<3,0$ $\mathrm{NMP} / \mathrm{g}$ de coliformes totais. O congelamento lento sem vácuo, no $37^{\circ}$ de armazenamento, manteve o referido resultado $(<3,0 \mathrm{NMP} / \mathrm{g})$. Nos demais tratamentos, maiores valores foram observados, 
sendo $7 \mathrm{NMP} / \mathrm{g}$ para congelamento lento com vácuo, e $93 \mathrm{NMP} / \mathrm{g}$ e $150 \mathrm{NMP} / \mathrm{g}$ quando armazenados sob congelamento rápido com vácuo e sem vácuo, respectivamente. Estes valores mais elevados podem ser resultantes de manipulação inadequada da amostra.

A legislação da ANVISA, resolução RDC n ${ }^{\circ} 12$ de 02 de janeiro de 2001, não estabelece parâmetros para a pesquisa dessas bactérias em alimentos prontos para o consumo.
Com base na análise de coliformes termotolerantes, ressalta-se o último dia de análise, 37 dias de armazenamento refrigerado após descongelamento, sob congelamento rápido sem vácuo, que apresentou crescimento dessa bactéria no valor de $7 \mathrm{NMP} / \mathrm{g}$. Nos demais dias, o guacamole apresentou resultados $<3,0 \mathrm{NMP} / \mathrm{g}$, caracterizando-o como produto estável para o consumo, visto que a $\mathrm{RDC} \mathrm{n}^{\circ} 12$ tolera a presença dessa bactéria até $102 \mathrm{NMP} / \mathrm{g}$.

Tabela 3. Contagem de coliformes totais e termotolerantes UFC/g do guacamole.

\begin{tabular}{|c|c|c|c|}
\hline Tratamentos & Tempo(dias) & Coliformes (NMP/G) Totais & Coliformes(NMP/G)Termotolerantes \\
\hline Controle & 0 & $>1.100$ & $<3,0$ \\
\hline \multicolumn{4}{|l|}{ Congelamento } \\
\hline \multirow[t]{4}{*}{ lento com vácuo } & 5 & 9 & $<3,0$ \\
\hline & 7 & 9 & $<3,0$ \\
\hline & 30 & $<3,0$ & $<3,0$ \\
\hline & 37 & 7 & $<3,0$ \\
\hline \multicolumn{4}{|l|}{ Congelamento } \\
\hline \multirow[t]{4}{*}{ lento sem vácuo } & 5 & 7 & $<3,0$ \\
\hline & 7 & 4 & $<3,0$ \\
\hline & 30 & $<3,0$ & $<3,0$ \\
\hline & 37 & $<3,0$ & $<3,0$ \\
\hline \multicolumn{4}{|l|}{ Congelamento } \\
\hline \multirow[t]{4}{*}{ rápido com vácuo } & 5 & 9 & $<3,0$ \\
\hline & 7 & $<3,0$ & $<3,0$ \\
\hline & 30 & $<3,0$ & $<3,0$ \\
\hline & 37 & 93 & $<3,0$ \\
\hline \multicolumn{4}{|l|}{ Congelamento } \\
\hline \multirow[t]{4}{*}{ rápido sem vácuo } & 5 & 4 & $<3,0$ \\
\hline & 7 & $<3,0$ & $<3,0$ \\
\hline & 30 & $<3,0$ & $<3,0$ \\
\hline & 37 & 150 & 7 \\
\hline
\end{tabular}


Arvizu-Medrano, Iturriaga e Escartín (2001) relataram que a polpa de abacate, quando submetida à refrigeração, diminui consideravelmente $o$ crescimento de E. coli, bactéria indicadora de contaminação fecal. Os mesmos autores encontraram E. coli em 29 amostras coletadas de vendedores de rua no México.

No presente estudo, o guacamole encontra-se em acordo com a resolução vigente da ANVISA, que estabelece até 102 UFC/g para a presença dessa bactéria em alimentos prontos para o consumo.

Salmonella sp. não foi detectada. Esses resultados se encontram de acordo com ArvizuMedrano, Iturriaga e Escartín (2001), que do mesmo modo relataram a ausência dessa bactéria nas amostras coletadas de polpa de abacate. Sendo assim, o produto se encontra em acordo com a RDC 12, que estabelece ausência de Salmonella em 25g de produto analisado.

Quanto à pesquisa de Staphylococcus coagulase positiva, no presente estudo o guacamole apresentou resultados $<100 \mathrm{UFC} / \mathrm{g}$ em todos os tratamentos e dias de armazenamento, assim como já relatado por Simon (2010) ao quantificar essa bactéria em amostras de guacamole sem presença de aditivos, estando de acordo com a RDC 12. Entretanto, Arvizu-Medrano, Iturriaga e Escartín (2001) verificaram a presença dessa bactéria em $6,7 \%$ das amostras de polpa de abacate, as quais apontaram resultados entre 105 e $106 \mathrm{UFC/g}$.

Neste trabalho, também foi realizada a pesquisa de Bacillus cereus. Este microrganismo não apresentou crescimento no produto. Também não houve crescimento de bactérias mesófilas, psicrotróficas, bolores e leveduras. Similarmente, Simon et al. (2010), ao pesquisar tais bactérias em guacamole conservado pelo frio sem presença de aditivos, também observou que estes microorganismos não cresceram durante os prazos de armazenamento congelado ou refrigerado.

As pesquisas de bactérias mesófilas e psicrotróficas não constam na RDC 12 de 02 de janeiro de 2001, entretanto, elas foram realizadas por se tratar de pesquisa comercial do produto.

De modo geral, as amostras foram microbiologicamente estáveis com as embalagens utilizadas, com e sem vácuo e ao longo do período de armazenamento considerado.

A adição de ácido ascórbico foi eficiente na conservação do guacamole armazenado sob congelamento lento ou rápido. Após descongelamento das amostras e armazenamento sob refrigeração, a qualidade do produto é negativamente alterada quanto aos parâmetros bioquímicos e sensoriais.

\section{Agradecimentos}

À empresa Jaguacy (Bauru-SP) pelo apoio e participação nas pesquisas, à Fundação de Apoio a Pesquisa no Estado de São Paulo (FAPESP) e CAPES.

\section{Referências}

AGÊNCIANACIONAL DE VIGILÂNCIA SANITÁRIA - ANVISA. Resolução - RDC n ${ }^{\circ} 12$, de 2 de janeiro de 2001. Disponível em: <www.anvisa.gov.br/legis/ resol/12_01rdc.htm. . . Acesso em: 01 fev. 2008.

AMERICAN PUBLIC HEALTH ASSOCIATION APHA. Compendium of methods for the icrobiological examination of foods. D. C., Washington: Apha, 2001. v. 4, $1219 \mathrm{p}$.

ARAÚJO, J. M. A. Química de alimentos: teoria e prática. 3. ed. Viçosa: UFV, 2006.

ARVIZU-MEDRANO, S. M.; ITURRIAGA, M. H.; ESCARTÍN, E. F. Indicator and pathogenic bactéria in guacamole and their behavior in avocado pulp. Journal of Food Safety, Querato, México, v. 21, p. 233-241, 2001.

BARROSO, L. P.; ARTES, R. Análise multivariada. In: SIMPÓSIO DE ESTATÍSTICA APLICADA A EXPERIMENTAÇÃO, 10., REUNIÃO ANUAL DA REGIÃO BRASILEIRA DA SOCIEDADE INTERNACIONAL DE BIOMETRIA, 48., 2003, Lavras. Anais... Lavras: URL, 2003. 151 p.

BEN-ET, G.; DOLEV, A.; TATARSK, D. Compounds 
contributing to heat-induced bitter off-flavor in avocado. Journal of Food Science, Chicago, v. 38, p. 546-547, 1973.

CANO, M. P.; ANCOS, B.; MANTALLANA, M. C.; CAMARA, N.; REGLERO, G.; TABEA, J. Differences among Spanish and Latin American banana cultivars: morphological, chemical and sensory characteristics. Food Chemistry, Banking, v. 59, n. 3, p. 411-419, 1997.

CHAVES, J. B. P.; SPROESSER, R. L. Práticas de laboratório de análise sensorial de alimentos e bebidas. Viçosa: UFV, 1999. (Cadernos Didáticos, 66).

DAIUTO, E. R.; VIEITES, R. L.; TACONELLI, C.; GOLÇALVES, A. de. F.; PIVETTA, P. R; SIMON, J. W. Avaliação sensorial do guacamole conservado pelo frio. Alimentos e Nutrição, Araraquara, v. 18, n. 34, p. 405412, out./dez. 2007.

GUZMÁN, G. R.; DORANTES, A. L.; HERNADEZ, U. H.; ORTIZ, A.; MORA, E. R. Effect of zinc and copper chloride on the color of avocado puree heated with microwaves. Innovative Food Science and Emerging Technologies, Holanda, v. 3, n. 7, p. 47-53. 2002.

LIMA, G. P. P. Efeito do cálcio sobre o teor de poliaminas $e$ atividade da peroxidase e redutase no nitrato em calos de arroz (Oriza sativa. L. cv. IAA 4440). 1994. Tese (Doutorado em Ciências Biológicas) - Instituto de Biociências. Universidade Estadual de São Paulo, Botucatu.

LÓPEZ-MALO, A.; PALOU, E.; BARBOSA, G.; WELTI, J.; SWANSON, B. Polyphenoloxidase activity and color changes during of high hydrostatic pressure treated avocado puree. Food Research International, Oxford, v. 31, p. 549-56,1998.

MARTIN, Z. J. Processamento: produtos, características e utilização. In: TEIXEIRA, C. G.; BLEINROTH, E. W.; CASTRO, J. V. de; MARTIN, Z. J. de; TANGO, J. S.; TURATTI, J. M., LEITE, R. S. S. F.; GARCIA, A. E. (Ed.). Abacate: cultura, matéria prima, processamento e aspectos econômicos. 2. ed. Campinas: ITAL, 1991. p. 148-155.

PALOU, E.; HÉRNANDEZ-SALGADO, C.; LOPEZMALO, A.; BARBOSA-CANÓVAS, G. V.; SWANSON, B. G.; WELT-CHANES, J. High pressure-processed guacamole. Innovative. Food Science and Emerging Technologies, Holanda, v. 1, p. 69-75, 2000.

PALOU, E.; LÓPEZ-MALO, A.; BARBOSACANOVAS, G. V.; WELTI-CHANES, J.; SWANSON, B. G. Polyphenoloxidase activity and color of blanched and high hydrostatic pressure treated banana puree. Journal of Food Science, Chicago, v. 64, p. 42-45, 1999.
R Development Core Team (2007). R: A language and environment for statistical computing. R Foundation for Statistical Computing, Vienna, Austria. Disponível em: $<$ http://www.R-project.org>. Acesso em: 15 set. 2007.

SIMON, J. W.; DAIUTO, E. R.; VIEITES, R. L.; RALL, V. L. M. Avaliação microbiológica e sensorial de guacamole conservado pelo frio. Revista Higiene Alimentar, São Paulo, v. 24, n. 180/181, p. 108-115, jan./ fev. 2010.

SON, S.; MOON, K.; LEE, C. Kinetic study of oxalic acid inhibition on enzymatic browning. Journal of Agricultural and Food Chemistry, Easton, v. 48, p. 20712074, 2000.

STEPHENS, T. S.; LIME, B. J.; GRIFFITHS, F. P. The effect of thickening agents in reducing the watery sepration of frozen and thawed guacamole products. Proceedings of the Rio Grande Valley Horticultural Society, Texas, v. 12, p. 81-87, 1958. 
